\title{
Clearance gap flow: extended pneumatic measurements and simulations by discontinuous Galerkin finite element method
}

\author{
Jindřich Hála ${ }^{1, a}$, Martin Luxa ${ }^{1}$, Ondřej Bublík ${ }^{2}$, Helena Prausová ${ }^{2}$, and Jan Vimmr ${ }^{2}$ \\ 1 Institute of Thermomechanics AS CR, v.v.i., Academy of Sciences of the Czech Republic, Prague, Czech Republic \\ 2 NTIS - New Technologies for the Information Society, Faculty of Applied Sciences, University of West Bohemia, Pilsen, \\ Czech Republic
}

\begin{abstract}
In the present paper, new results of measurements of the compressible viscous fluid flow in narrow channels with parallel walls under the conditions of aerodynamic choking are presented. Investigation was carried out using the improved test section with enhanced capability to accurately set the parallelism of the channel walls. The measurements were performed for the channels of the dimensions: length $100 \mathrm{~mm}$, width $100 \mathrm{~mm}$ and for various heights in the range from $0.5 \mathrm{~mm}$ to $4 \mathrm{~mm}$. The results in the form of distribution of the static pressure along the channel axis including the detailed study of the influence of the deviation from parallelism of the channel walls are compared with previous measurements and with numerical simulations performed using an in-house code based on Favre averaged system of Navier-Stokes equations completed with turbulence model of Spalart and Allmaras and a modification of production term according to Langtry and Sjolander. The spatial discretization of the governing equations is performed using the discontinuous Galerkin finite element method which ensures high order spatial accuracy of the numerical solution.
\end{abstract}

\section{Introduction}

The need to study compressible viscous fluid flow in narrow channels emerges from the occurrence of such flows in many engineering applications, for example in clearance gaps of the screw type compressors, during tip leakage flows in steam and gas turbine stages without shroud and in clearance gaps of the control valves of steam turbines. Such a widespread engineering application calls for need to explain and predict dissipative phenomena influencing thermodynamic losses and thus the efficiency of these machines.

The study follows earlier efforts of the authors, [1], and extends their most recent work, [2]. Similarly as in the case of previous experiments, the channel of the height varying from 0.5 to $4 \mathrm{~mm}$, length $100 \mathrm{~mm}$ and width $100 \mathrm{~mm}$ was chosen as the simplified model. However, the test section was improved to allow more accurate setting of the parallelism of the channel walls, because previous experiments showed that even small deviation have significant influence on the pressure distribution along the channel.

The aim of this study was to prove the increased capabilities of the new test section, namely to study the reproducibility of the experiments, and gather more reliable data for comparison with the results of numerical simulations, performed by an implicit solver based on the discontinuous Galerkin finite element method.

\footnotetext{
a e-mail: jindrich.hala@gmail.com
}

\section{Experiment}

\subsection{Settings of the experiment}

The experiments were carried out in the Aerodynamics Laboratory of the Institute of Thermomechanics AS CR, v.v.i. in Nový Knín. Scheme of the settings of the experiment can be seen in the Figure 1. The channel is formed by two parallel polished steel plates with the rounded leading edges (Figure 2) and by two glass windows. Unlike the test section used for the previous measurements, the current one was designed in order to have much higher stiffness to prevent the deformation of the walls. Moreover, eight adjusting screws were added (one in each corner of the lower and upper channel walls) to allow more precise setting of the parallelism of the gap.

During the measurement, the air continues from the laboratory through the channel and goes to the settling chamber in which the required back pressure $p_{b}$ is maintained using the control nozzle downstream the settling chamber. Then the air continues through the pipes to the vacuum storage.

\subsection{Methods of measurements}

The upper wall of the channel was equipped with the static pressure taps, location of which can be seen in the Figure 3. For the pressure data acquisition, two 16 channel pressure scanners PSI 9116 were used. The scanner integrates 16 silicon piezoresistive pressure sensors and microprocessor that performs a correction for sensor zero, span, linearity and thermal errors. The manufacturer guarantees system accuracy of up to $\pm 0.05 \%$ of the full scale. The connection between the pressure scanners and pressure taps was 


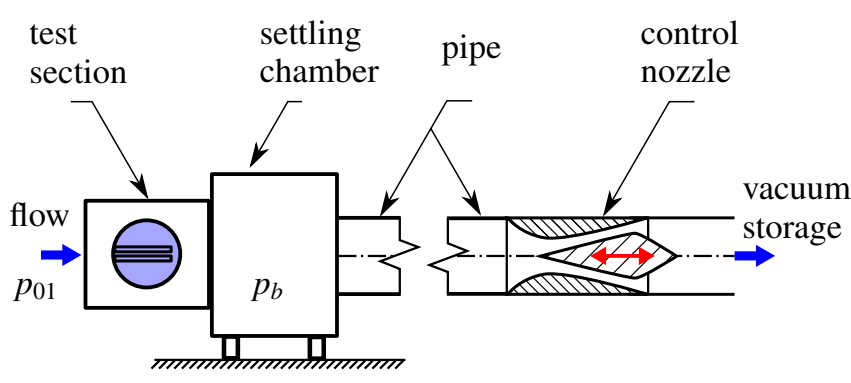

Fig. 1: Scheme of the set up of the experiment.

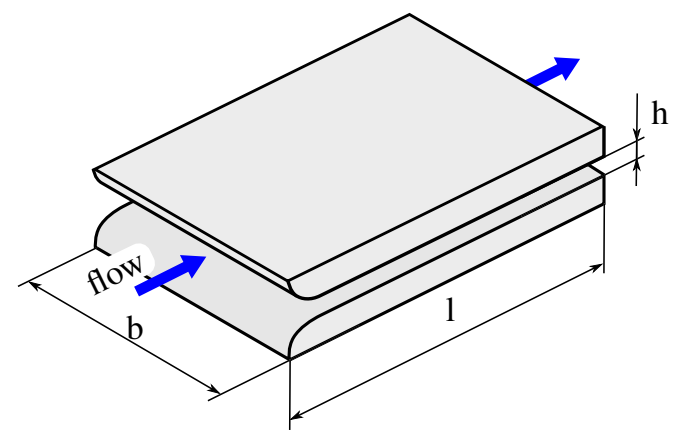

Fig. 2: Schematic view of the channel formed by two steel plates with marked main dimensions $l, b$ and $h$ (windows serving as a side walls are not depicted).

realised using the plastic tubes of diameter $0.5 \mathrm{~mm}$ and length up to $0.5 \mathrm{~m}$.

To obtain more information about the flow field in the channel, the optical methods were incorporated in the form of Mach-Zehnder interferometry, [2], [3]. This method allows to examine compressible fluid flow in the channel without intrusion of any probe, however, due to the small dimensions of the channel, quantitative evaluation of the interferograms is possible only in the case of gaps from height approximately $h=2 \mathrm{~mm}$. Nevertheless, even for smaller gaps, flow field near the exit of the channel can be depicted in terms of isolines of index of refraction and used for qualitative assessment of the numerical simulation.

\subsection{Results of the experiment}

The experimental investigation was carried out for a channel of the height $h=0.5,1,1.5,2,2.5,3$ and $4 \mathrm{~mm}$. Both optical and pneumatic measurements were performed for pressure ratios (ratio of the back pressure $p_{b}$ to stagnation pressure $\left.p_{01}\right) \pi=0.189,0.195,0.308,0.375$ and 0.457 . Such pressure ratios were chosen in order to reach aerodynamic choking in the channel.

In the Figure 5 the comparison of the distribution of pressure ratio $\frac{p_{i}}{p_{01}}$ (ratio of the static pressure in each measuring point $p_{i}$ to stagnation pressure $p_{01}$ ) along the channel axis for $\pi=0.188$, obtained using pneumatic methods, can be seen for all investigated gap heights. For the gap heights up to $3 \mathrm{~mm}$, we can observe steeper gradient of the pressure near the channel inlet than in the middle part of the channel. This might be caused by the abrupt change in the surface curvature on the interface of the rounded inlet and straight part of the channel, that is disturbing flow properties downstream such a point. In the middle part of

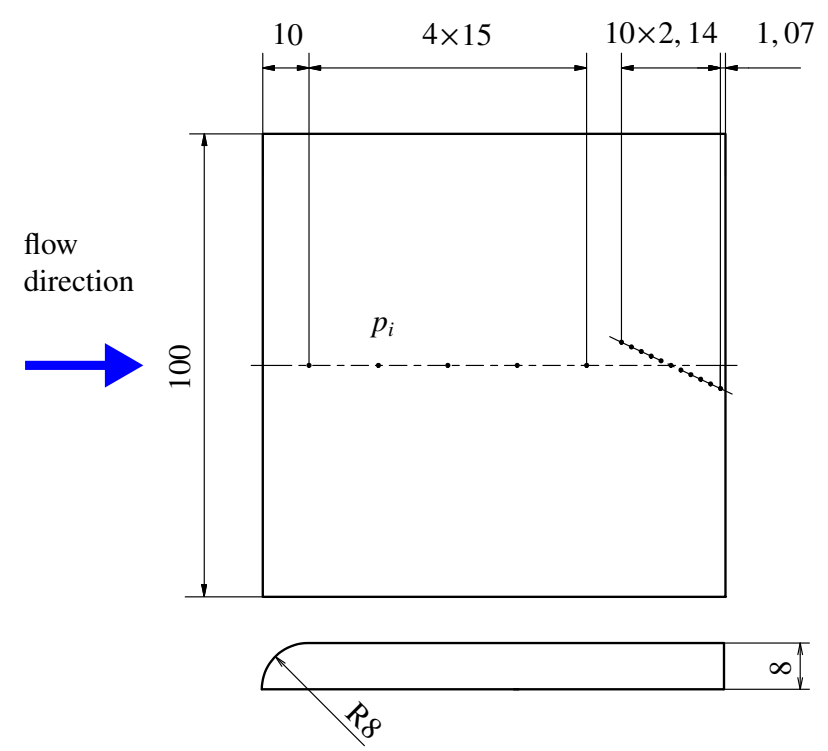

detail of the pressure tap

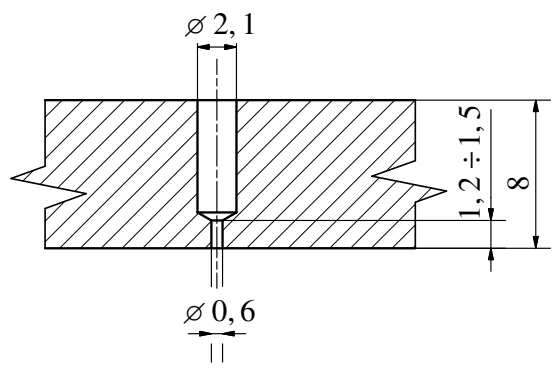

Fig. 3: Scheme of the upper wall of the minichannel of the lenght $100 \mathrm{~mm}$ with plotted positions of pressure taps and a detail view of the pressure tap.

the channel, the pressure distribution is linear and the slope of the line depends on the gap height, so that for narrow gap, the gradient of the pressure is higher due to more significant influence of the friction. With increasing gap height, the pressure gradient decreases towards the pressure distribution in the theoretical channel with negligible friction (zero pressure gradient). Following the linear part, there we can see significant pressure drop starting approximately at $80 \%$ of the channel length, where the flow accelerates towards sonic speed in the vicinity of the exit.

Pressure distributions for all other pressure ratios $\pi$ are quite similar due to the phenomenon of aerodynamic choking. The only difference could be observed in the flow structure downstream the exit of the channel (Figure 4).

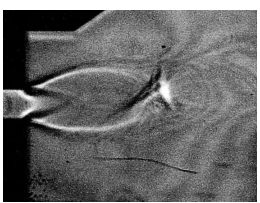

(a)

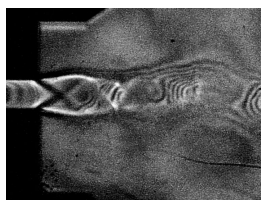

(b)

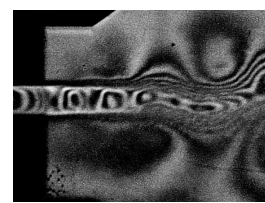

(c)
Fig. 4: Interferograms of the flow field downstream the exit of the channel of the height $4 \mathrm{~mm}$ for pressure ratios $\pi=$ 0.194 (a), $\pi=0.308$ (b) and $\pi=0.458$ (c) 


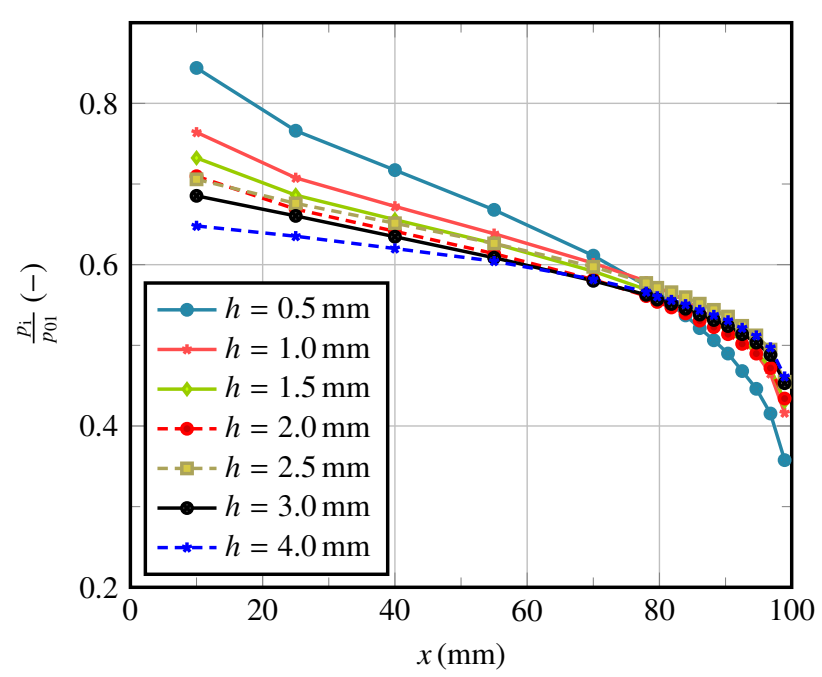

Fig. 5: Distribution of the pressure ratio $\frac{p_{i}}{p_{01}}$ along the minichannel axis for various channel heights obtained from pneumatic measurements, $\pi=0.188$.

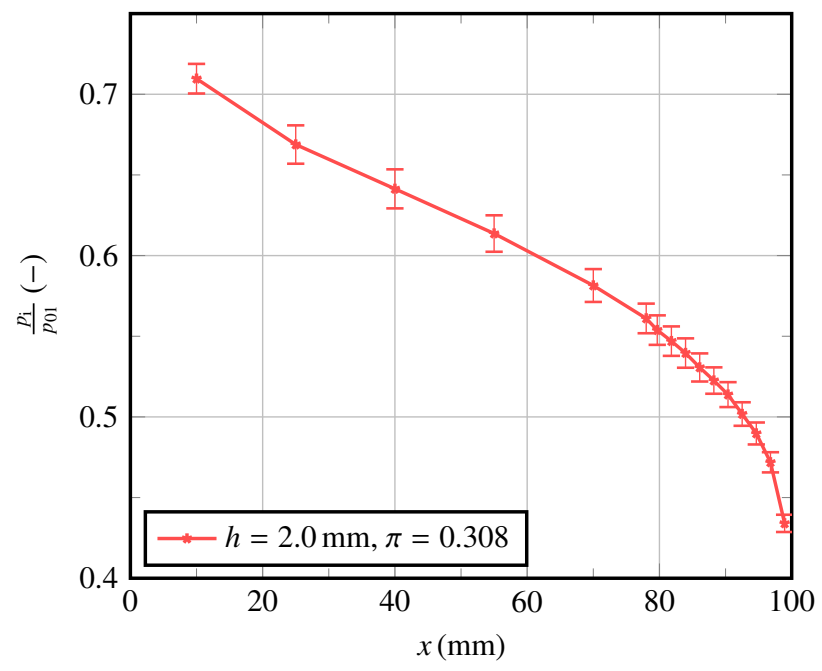

Fig. 6: Mean value of the ratio of the static pressure and stagnation pressure $p_{01}$ along the channel axis for pressure ratio $\pi=0.308$, obtained using 10 pneumatic measurements for channel height $h=2 \mathrm{~mm}$, each with different adjustment. Error bars indicate the value of standard deviation.

The other part of the experiment was focused on the reproducibility of the results. For this purpose, the gap of the height $2 \mathrm{~mm}$ was used. Then 10 measurements were performed, each for slightly different adjustment of the adjustment screws. Deviation from the parallelism was measured in the locations of channel corners using feeler gauges and did not exceed $0.1 \mathrm{~mm}(5 \%)$. Both combinations of slightly converging and diverging channels were explored as well as channels with slight transverse slope. Results can be seen in the plot in the Figure 6, where the mean value of mentioned 10 measurements is plotted together with error bars indicating the value of standard deviation.

\section{Numerical simulation}

Numerical simulations of the air flow through the considered narrow channel were performed to further explore compressible fluid flow properties in this type of channels and demonstrate suitability of the in-house code based on the discontinuous Galerkin finite element method for that kind of computations. The width of the channel enables to simplify the geometry for numerical simulation into two dimensions.

\subsection{Mathematical model}

Mathematical model used to describe the problem is based on Favre-averaged system of Navier-Stokes equations written in dimensionless form as

$$
\begin{aligned}
& \frac{\partial \bar{\varrho}}{\partial t}+\frac{\partial\left(\bar{\varrho} \tilde{u}_{j}\right)}{\partial x_{j}}=0, \\
& \frac{\partial}{\partial t}\left(\bar{\varrho} \tilde{u}_{i}\right)+\frac{\partial}{\partial x_{j}}\left(\bar{\varrho} \tilde{u}_{i} \tilde{u}_{j}+\bar{p} \delta_{i j}\right)=\frac{1}{R e} \frac{\partial}{\partial x_{j}}\left(\tilde{t}_{i j}+\tau_{i j}\right) \text {, } \\
& \frac{\partial}{\partial t}(\bar{\varrho} \tilde{e})+\frac{\partial}{\partial x_{j}}\left(\bar{\varrho} \tilde{e} \tilde{u}_{j}+\bar{p} \tilde{u}_{j}\right)=\frac{1}{R e} \frac{\partial}{\partial x_{j}}\left[\left(\tilde{t}_{i j}+\tau_{i j}\right) \tilde{u}_{i}+\right. \\
& \left.+\frac{\kappa}{\kappa-1}\left(\frac{\mu}{P r}+\frac{\mu_{t}}{P r_{t}}\right) \frac{\partial}{\partial x_{j}}\left(\frac{\bar{p}}{\bar{\varrho}}\right)\right],
\end{aligned}
$$

where $\bar{\varrho}, \tilde{u}_{i}, \bar{p}, \tilde{e}$ are dimensionless mass- or time-averaged values of density, velocity, pressure and energy. Massaveraged stress tensor and Reynolds stress tensor are given by $\tilde{t}_{i j}=2 \mu \bar{S}_{i j}, \tau_{i j}=2 \mu_{t} \bar{S}_{i j}$, where $\bar{S}_{i j}=\frac{1}{2}\left(\frac{\partial \tilde{u}_{i}}{\partial x_{j}}+\frac{\partial \tilde{u}_{j}}{\partial x_{i}}\right)-$ $\frac{1}{3} \delta_{i j} \frac{\partial \tilde{u}_{k}}{\partial x_{k}}$.

A one-equation turbulence model of Spalart and Allmaras [5] is implemented to complete the Favre-averaged system of Navier-Stokes equations. It is given by transport equation for eddy viscosity $\tilde{v}$ written as

$$
\begin{aligned}
& \frac{\partial(\varrho \tilde{\varrho} \tilde{v})}{\partial t}+\frac{\partial\left(\bar{\varrho} \tilde{v} \tilde{u}_{j}\right)}{\partial x_{j}}=\frac{1}{\operatorname{Re}} \frac{1}{\sigma}\left[\frac{\partial}{\partial x_{j}}\left((\mu+\bar{\varrho} \tilde{v}) \frac{\partial \tilde{v}}{\partial x_{j}}\right)+\right. \\
& \left.+\bar{\varrho} c_{b 2} \frac{\partial \tilde{v}}{\partial x_{j}} \frac{\partial \tilde{v}}{\partial x_{j}}\right]+P T M c_{b 1} \bar{\varrho} \tilde{S} \tilde{v}-\frac{1}{R e} c_{w 1} f_{w} \frac{1}{\bar{\varrho}}\left(\frac{\bar{\varrho} \tilde{v}}{D}\right)^{2}
\end{aligned}
$$

which is completed by a set of auxiliary relations

$$
\begin{aligned}
& \mu_{t}=\bar{\varrho} \tilde{v} f_{v 1}, \\
& \tilde{S}=\bar{\Omega}+\frac{1}{\operatorname{Re} \frac{\tilde{v}}{\kappa^{2} D^{2}} f_{v 2},} \\
& f_{w}=g\left(\frac{1+c_{w 3}^{6}}{g^{6}+c_{w 3}^{6}}\right)^{\frac{1}{6}}, \\
& g=r+c_{w 2}\left(r^{6}-r\right), \\
& r=\min \left(\frac{\tilde{v}}{\operatorname{Re} \tilde{S} \kappa^{2} D^{2}}, 10\right), \\
& f_{v 1}=\frac{\chi^{3}}{\chi^{3}+c_{v 1}^{3}}, \quad \chi=\frac{\tilde{v}}{v}, \\
& f_{v 2}=1-\frac{\chi}{1+\chi f_{v 1}}
\end{aligned}
$$

and constants $c_{b 1}=0.1355, c_{b 2}=0.622, \sigma=\frac{2}{3}, \kappa=$ $0.41, c_{w 1}=\frac{c_{b 1}}{\kappa^{2}}+\frac{1+c_{b 2}}{\sigma}, c_{w 2}=0.3, c_{w 3}=2.0, c_{v 1}=7.1$, 
where $\Omega$ is the vorticity magnitude and $D$ is the distance to the nearest wall.

The governing equations are then completed by a transitional model developed by Langtry and Sjolander (2002), [5]. Principle of this model is to restrict the production of turbulent kinetic energy in $k-\omega$ model by multiplying it by term PTM (production term modification) with values from 0 to 1 . In the presented work the PTM term is applied to the eddy viscosity production term in transport equation (2). Value of PTM is computed from flow field variables and enables to switch gradually from laminar to turbulent flow regime. The model is based on the vorticity Reynolds number

$$
R e_{v}=\frac{\varrho D^{2}}{\mu} \Omega
$$

The PTM term takes the form of

$$
P T M=1-P T M_{1} F_{3},
$$

where function $F_{3}$ is defined as

$$
F_{3}=\exp \left[-\left(\frac{R_{T}}{6.5}\right)^{4}\right]
$$

and viscosity ratio is simply $R_{T}=\frac{\mu_{t}}{\mu} . P T M_{1}$ term is given by two following polynomials

$$
P T M_{1}=\left\{\begin{array}{c}
1-\left[\begin{array}{c}
(3.82 E-04) R e_{v}- \\
(3.94 E-07) R e_{v}^{2} \\
(1.43 E-10) R e_{v}^{3}
\end{array}\right], \quad R e_{v}<1000 \\
1-\left[0.12+(1.0 E-05) R e_{v}\right], R e_{v} \geq 1000 .
\end{array}\right.
$$

System of averaged Navier-Stokes equations (1) and the transport equation of turbulence model (2) can be written together as

$$
\begin{aligned}
& \frac{\partial w}{\partial t}+\sum_{j=1}^{2} \frac{\partial}{\partial x_{j}} \boldsymbol{f}_{j}(\boldsymbol{w})= \\
&=\frac{1}{\operatorname{Re}} \sum_{j=1}^{2} \frac{\partial}{\partial x_{j}} \boldsymbol{f}_{j}^{v}(\boldsymbol{w}, \nabla \boldsymbol{w})+\boldsymbol{p}(\boldsymbol{w}, \nabla \boldsymbol{w}),
\end{aligned}
$$

where $\boldsymbol{w}$ is a vector of conservative variables, $\boldsymbol{f}_{j}(\boldsymbol{w})$ are the inviscid numerical fluxes, $\boldsymbol{f}_{j}^{v}(\boldsymbol{w}, \nabla \boldsymbol{w})$ are the viscid numerical fluxes and $\boldsymbol{p}(\boldsymbol{w}, \nabla \boldsymbol{w})$ is a production term. The vectors are written as

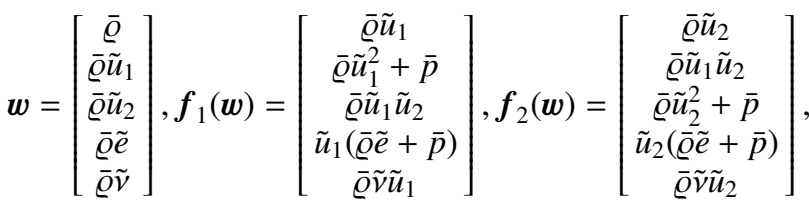

$$
\begin{aligned}
& \boldsymbol{f}_{1}^{v}(\boldsymbol{w}, \nabla \boldsymbol{w})=\left[\begin{array}{c}
0 \\
\tilde{t}_{11}+\tau_{11} \\
\tilde{t}_{12}+\tau_{12} \\
\sum_{i=1}^{2} \tilde{u}_{i}\left(\tilde{t}_{i 1}+\tau_{i 1}\right)+\frac{\kappa}{\kappa-1}\left(\frac{\mu}{P r}+\frac{\mu_{t}}{P r_{t}}\right) \frac{\partial}{\partial x_{1}}\left(\frac{\bar{p}}{\bar{\varrho}}\right) \\
\frac{1}{\sigma}(\mu+\varrho \bar{\varrho}) \frac{\partial \tilde{v}}{\partial x_{1}}
\end{array}\right], \\
& \boldsymbol{f}_{2}^{v}(\boldsymbol{w}, \nabla \boldsymbol{w})=\left[\begin{array}{c}
0 \\
\tilde{t}_{12}+\tau_{12} \\
\tilde{t}_{22}+\tau_{22} \\
\sum_{i=1}^{2} \tilde{u}_{i}\left(\tilde{t}_{i 2}+\tau_{i 2}\right)+\frac{\kappa}{\kappa-1}\left(\frac{\mu}{P r}+\frac{\mu_{t}}{P r_{t}}\right) \frac{\partial}{\partial x_{2}}\left(\frac{\bar{p}}{\bar{\varrho}}\right) \\
\frac{1}{\sigma}(\mu+\varrho \bar{\varrho}) \frac{\partial \tilde{v}}{\partial x_{2}}
\end{array}\right],
\end{aligned}
$$

$$
\boldsymbol{p}(\boldsymbol{w}, \nabla \boldsymbol{w})=\left[\begin{array}{c}
0 \\
0 \\
0 \\
0 \\
\frac{1}{R e} \frac{1}{\sigma} \bar{\varrho} c_{b 2}\left(\frac{\partial \tilde{v}}{\partial x_{1}} \frac{\partial \tilde{v}}{\partial x_{1}}+\frac{\partial \tilde{v}}{\partial x_{2}} \frac{\partial \tilde{v}}{\partial x_{2}}\right)+ \\
P T M c_{b 1} \bar{\varrho} \tilde{S} \tilde{v}-\frac{1}{R e} c_{w 1} f_{w} \frac{1}{\bar{\varrho}}\left(\frac{\bar{\varrho} \tilde{v}}{D}\right)^{2}
\end{array}\right] .
$$

\subsection{Discretization}

The spatial discretization of equations (3) is performed using the discontinuous Galerkin finite element method (DGFEM), [6], [7], [8].

Let $\mathcal{T}_{h}=\left\{\Omega_{k}: \Omega_{k} \in \Omega, k=1, \ldots, K\right\}$ be a triangulation of the computational domain $\Omega \in \mathcal{R}^{2}$ and $\Gamma=\Gamma^{\text {int }} \cup \Gamma^{b}$ be the set of inner $\Gamma^{\text {int }}$ and boundary $\Gamma^{b}$ edges. The solution of equations (3) is considered in the space of vector functions $\mathbf{S}_{h}=S_{h} \times S_{h} \times S_{h} \times S_{h}$, where

$$
\begin{array}{r}
S_{h}=\left\{v(\mathbf{x}): v(\mathbf{x}) \in \mathcal{P}^{q}\left(\Omega_{k}\right) \text { if } \mathbf{x} \in \Omega_{k},\right. \\
\left.v(\mathbf{x})=0 \text { if } \mathbf{x} \notin \Omega_{k}, \forall \Omega_{k} \in \mathcal{T}_{h}\right\}
\end{array}
$$

and $\mathcal{P}^{q}\left(\Omega_{k}\right)$ is the space of polynomials of $q$-th order at the most.

Multiplying the system of equations by a test function $\mathbf{v} \in \mathbf{S}_{h}$, integrating it over the element $\Omega_{k}$ and using the Green's theorem, the following integral identity is obtained

$$
\begin{gathered}
\int_{\Omega_{k}} \frac{\partial \boldsymbol{w}}{\partial t} \cdot \boldsymbol{v} \mathrm{d} \Omega-\int_{\Omega_{k}} \sum_{s=1}^{2} \boldsymbol{f}_{s}(\boldsymbol{w}) \cdot \frac{\partial \boldsymbol{v}}{\partial x_{s}} \mathrm{~d} \Omega+ \\
+\oint_{\partial \Omega_{k}} \sum_{s=1}^{2} \boldsymbol{f}_{s}(\boldsymbol{w}) n_{s} \cdot \boldsymbol{v} \mathrm{d} l=\frac{1}{\operatorname{Re}} \oint_{\partial \Omega_{k}} \sum_{s=1}^{2} \boldsymbol{f}_{s}^{v}(\boldsymbol{w}, \nabla \boldsymbol{w}) n_{s} \cdot \boldsymbol{v} \mathrm{d} l- \\
\frac{1}{\operatorname{Re}} \int_{\Omega_{k}} \sum_{s=1}^{2} \boldsymbol{f}_{s}^{v}(\boldsymbol{w}, \nabla \boldsymbol{w}) \cdot \frac{\partial \boldsymbol{v}}{\partial x_{s}} \mathrm{~d} \Omega+\int_{\Omega_{k}} \boldsymbol{p}(\boldsymbol{w}, \nabla \boldsymbol{w}) \cdot \boldsymbol{v} d \Omega .
\end{gathered}
$$

The key part of the method is an approximation of curve integrals. They are evaluated in the same way as it is done in the case of finite volume method. The inviscid flux is evaluated as

$$
\begin{gathered}
\sum_{s=1}^{2} \boldsymbol{f}_{s}(\boldsymbol{w}) n_{s} \approx \mathcal{H}\left(\boldsymbol{w}^{+}, \boldsymbol{w}^{-}\right), \text {on } \Gamma^{i n t} \in \partial \Omega, \\
\sum_{s=1}^{2} \boldsymbol{f}_{s}(\boldsymbol{w}) n_{s} \approx \mathcal{H}\left(\boldsymbol{w}^{b}, \boldsymbol{w}^{-}\right), \text {on } \Gamma^{b} \in \partial \Omega,
\end{gathered}
$$

where $\mathcal{H}$ is Lax-Friedrichs flux, [9], $\boldsymbol{n}=\left[n_{1}, n_{2}\right]$ is vector of outer normal, values $\boldsymbol{w}^{-}$resp. $\boldsymbol{w}^{+}$are the left resp. right limits of the vector $\boldsymbol{w}$ on the edge $\Gamma$ and $\boldsymbol{w}^{b}$ is a boundary value of $\boldsymbol{w}$ computed from boundary conditions.

Similarly, the value of viscous flux and the value of $w$ on the edge for gradient equation are evaluated using the appropriate numerical fluxes. In present paper, the approximation known as interior penalty (IP) method was chosen, [8].

Let $\boldsymbol{w}_{k}$ be a part of solution $\boldsymbol{w}$ corresponding to control element $\Omega_{k}$ and $\varphi_{k, i}$ be $i$-th basis function of the space $\mathcal{P}^{q}\left(\Omega_{k}\right)$. The $m$ components $w_{k}^{m}, m=1,2, \ldots 4$, of vector $\boldsymbol{w}_{k}$ can be expressed as a linear combination

$$
w_{k}^{m}=\sum_{i=1}^{q} w_{k, i}^{m}(t) \varphi_{k, i}(\boldsymbol{x}) .
$$


Inserting it into the integral identity and evaluating the volume and curve integrals using the Gauss integration rules of appropriate order, the following system of ordinary differential equations is obtained

$$
\mathbf{M} \frac{\mathrm{d} \mathbf{W}}{\mathrm{d} t}=\mathbf{R}(\mathbf{W}),
$$

where vector $\mathbf{W}$ consists of coefficients of linear combination (4) and $\mathbf{M}$ is a mass matrix. Linear Lagrange polynomials are chosen as the basis functions of the space $\mathcal{P}^{q}\left(\Omega_{k}\right)$.

Time integration of equation (5) is performed using the implicit backward Euler method in the form

$$
\left[\frac{\boldsymbol{M}}{\Delta t}-\frac{\partial \boldsymbol{R}}{\partial \boldsymbol{W}}\left(\boldsymbol{W}^{n}\right)\right] \Delta \boldsymbol{W}=\boldsymbol{R}\left(\boldsymbol{W}^{n}\right), \quad \boldsymbol{W}^{n+1}=\boldsymbol{W}^{n}+\Delta \boldsymbol{W}
$$

The system of linear equations (6) is solved iteratively using the GMRES method with block diagonal Jacobi preconditioner.

\subsection{Numerical results and comparison with experimental data}

Since the gap of the height $2 \mathrm{~mm}$ and pressure ratio $\pi=$ 0.308 was chosen to perform repeated measurements to explore reproducibility of the results (Figure 6), air flow for the same conditions was simulated in the first place. Distribution of static to stagnation pressure ratio along the axis channel is displayed in Figure 7. Very good agreement of the numerical and experimental results can be seen as the numerical data fit into the range of the standard deviation. The transitional model as presented above was used.

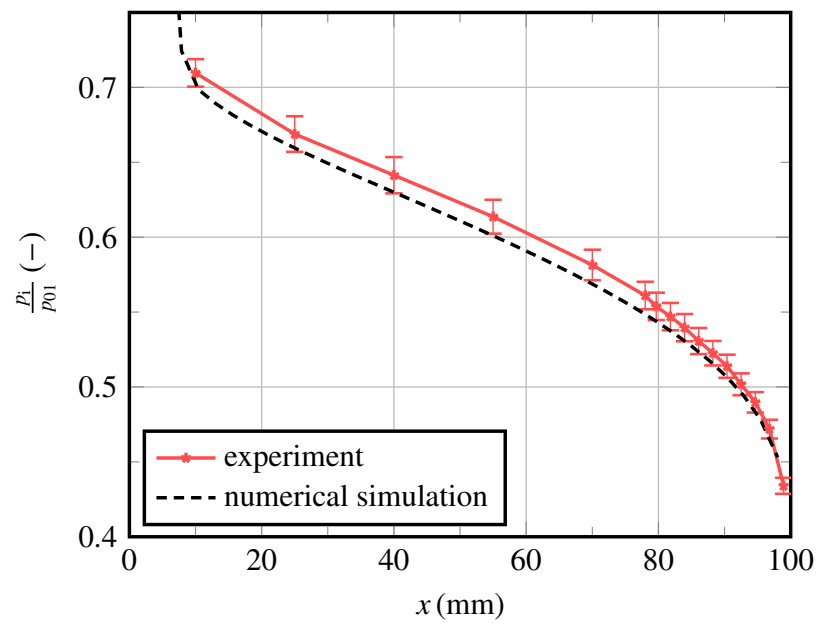

Fig. 7: Distribution of static to stagnation pressure ratio along the channel axis, $\mathrm{h}=2 \mathrm{~mm}, \pi=0.308$ : numerical and experimental results.

The aim of the numerical study is also to be able to compute properly the air flow behind the narrow channel. Qualitative comparison of computed density contour lines and the interferogram can be seen in Figures 8 and 9. The sequence of shock waves forming in the wake is captured well.

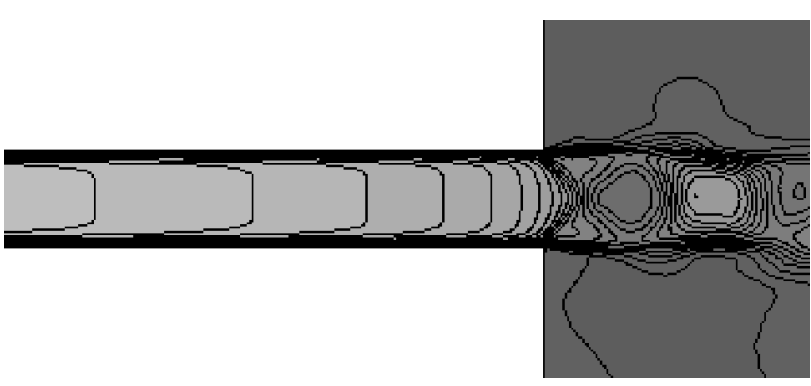

Fig. 8: Density isolines at the outflow, obtained from simulation with transitional model, $\mathrm{h}=2 \mathrm{~mm}, \pi=0.308$.

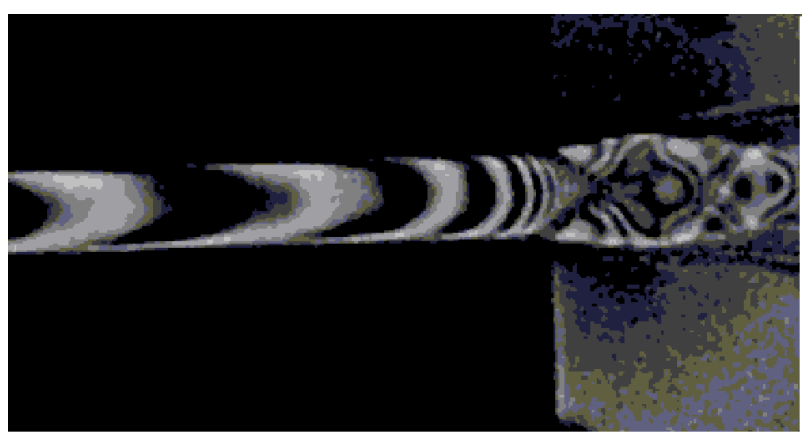

Fig. 9: Interferogram at the outflow, $\mathrm{h}=2 \mathrm{~mm}, \pi=0.308$.

Compressible flow in channels of the other heights was simulated as well. Distribution of static to stagnation pressure ratio along the axis channel of height $4 \mathrm{~mm}$ and pressure ratio $\pi=0.194$ is displayed in Figure 10. Laminar, fully turbulent and transitional numerical results can be seen in comparison with experimental data.

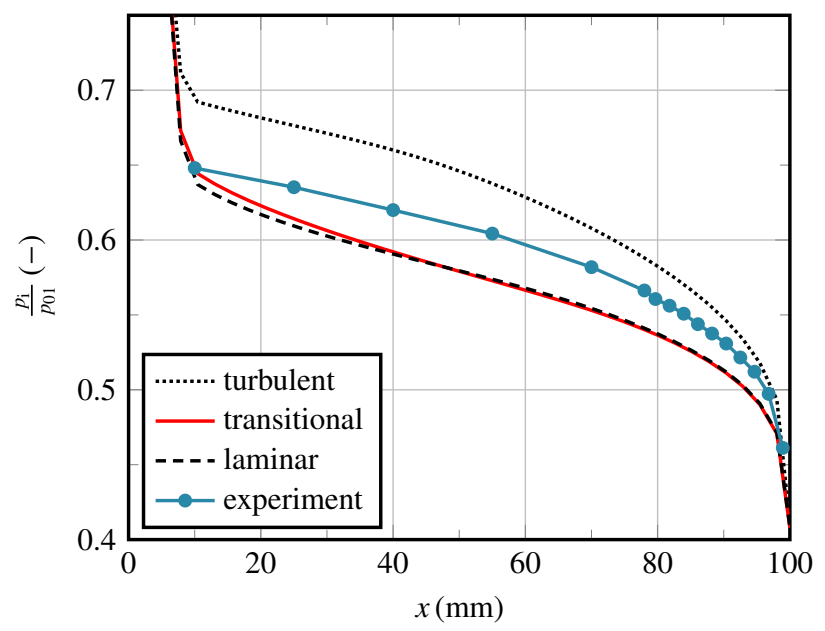

Fig. 10: Distribution of static to stagnation pressure ratio along the channel axis, $\mathrm{h}=4 \mathrm{~mm}, \pi=0.194$ : numerical and experimental results.

As in the case of $2 \mathrm{~mm}$ high channel, the air flow behind the channel of height $4 \mathrm{~mm}$ is also compared to experimental results, in Figures 11 and 12.

The good agreement in the pressure ratio at the beginning of the gap (position $\mathrm{x}=0.01 \mathrm{~m}$ ) in case of transitional modelling and the difference between experiment and sim- 


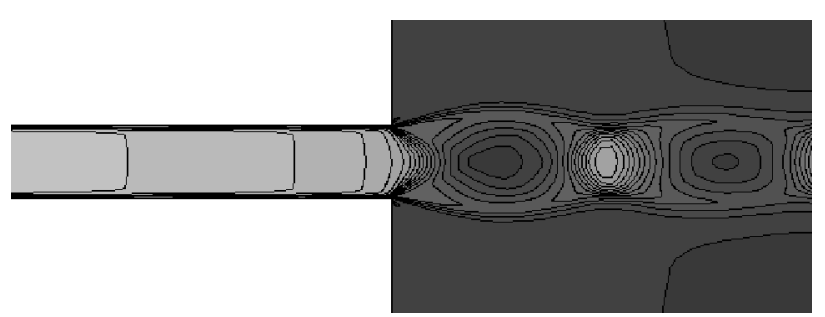

Fig. 11: Density isolines at the outflow, obtained from simulation with transitional model, $\mathrm{h}=4 \mathrm{~mm}, \pi=0.194$.

ulation further downstream indicate the need to more appropriately capture the transition from laminar to turbulent flow in the channel.

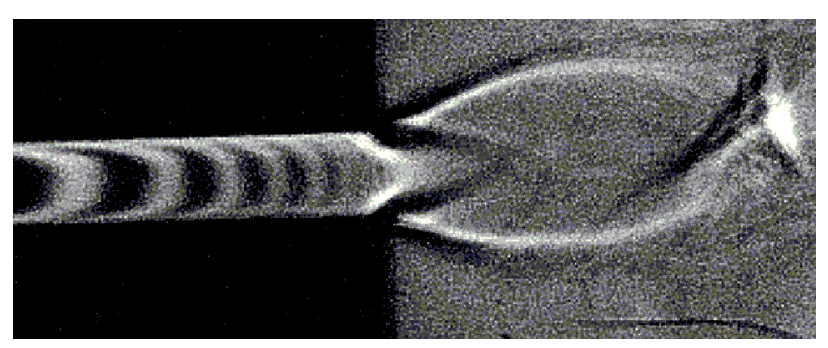

Fig. 12: Interferogram at the outflow, $h=4 \mathrm{~mm}, \pi=0.194$.

\subsection{Summary}

Viscous fluid flow in narrow gaps have been studied experimentally and the results were compared with numerical simulations. Improved test section proved to perform well during the experiments and allowed to gather new data for wider range of the gap heights. Moreover, the data obtained from reproduced measurements of the gap height $h=2 \mathrm{~mm}$ provided valuable data about the reproducibility of the measurements. Even though the results of the numerical simulations using transitional model of turbulence correspond well with experimental data, it must be said that the nature of the transition is still unexplored and will be subject to the following research as well as the study of wall friction.

Acknowledgement: Institutional support RVO: 61388998 is gratefully acknowledged. This publication was also supported by the project LO1506 of the Czech Ministry of Education, Youth and Sports.

\section{References}

1. Vimmr J., Klášterka H., Hajžman M., Luxa M., Dvořák R.: Journal of Thermal Science, 19(4), 289 -294 (2010).

2. Prausová H., Bublík O., Vimmr J., Luxa M., Hála J.: EPJ Web of Conferences 92, 02073 (2015).

3. M. Mokrý, Aerodynamic measurements using MachZehnder interferometer. Scientific Report Z-239/67. Institute of Thermomechnics AS CR, v.v.i., Prague (1967).

4. Spalart P. R., Allmaras S. R.: A One-Equation Turbulence model for Aerodynamic Flows. AIAA Paper 92439 (1992).
5. Langtry R. B., Sjolander S. A.: Prediction of Transition for Attached and Separated Shear Layers in Turbomachinery. AIAA Paper 2002-3641 (2002).

6. Reed W. H., Hill T. R.: Los Alamos Scientific Laboratory Report LA-UR-73-479 (1973).

7. Cockburn B., Shu C.-W.: Journal of Scientific Computing 16, 173 - 261 (2001).

8. Dolejší V., Holík M., Hozman J.: Journal of Computational Physics 230(11), 4176-4200 (2011).

9. Toro E. F.: Riemann Solvers and Numerical Methods for Fluid Dynamics, Springer, Heidelberg, pp. 325 (1999). 